\title{
Phase-space Fisher information of 2D gapped Dirac materials
}

\author{
Juan Carlos Bolívar ${ }^{1}$ - Ágnes Nagy ${ }^{2}$ Elvira Romera ${ }^{1}$
}

Received: 16 August 2018 / Accepted: 16 February 2019 / Published online: 26 February 2019

(c) The Author(s) 2019

\begin{abstract}
It is shown that phase-space Fisher information marks topological phase transitions in 2D Dirac gapped materials. The phase-space Fisher information determined for electrons and holes exhibit minimum at the charge neutrality points. Topological quantum numbers are defined to identify different phases utilizing combined information measures. Upper and lower bounds are derived for the phase-space Fisher information.
\end{abstract}

Keywords Phase-space Fisher information - Topological phase transitions · Dirac materials

\section{Introduction}

Information theoretical concepts have become very important in several fields of research. In this paper we focus on phase-space Fisher information (PSFI) [1-3] and explore how it describes topological phase transitions (TPT). Recently, there has been intense activity in studying Dirac materials. Here we focus on silicene, a $2 \mathrm{D}$ crystal of silicon having a low-buckled honeycomb arrangement. Perpendicularly to the silicene sheet, there is a shift in the two sublattices with a buckled height of $l=0.44 \AA$ ) due to large interatomic distances. It has a significant spin-orbit interaction giving rise to a small band gap opening at the Dirac point [4,5]. Silicene is a topological insulator (TI) [6], but it changes to a band insulator (BI) [7] due to the spin-orbit interaction and a

\footnotetext{
Ágnes Nagy

anagy@phys.unideb.hu

Juan Carlos Bolívar

jcbolivar@ugr.es

Elvira Romera

eromera@ugr.es

1 Departamento de Física Atómica, Molecular y Nuclear and Instituto Carlos I de Física Teórica y

Computacional, Universidad de Granada, Fuentenueva s/n , 18071 Granada, Spain

2 Department of Theoretical Physics, University of Debrecen, Debrecen 4002, Hungary
} 
transverse external field. 2D TI was theoretically predicted by Kane and Melle [8] and later was observed experimentally in mercury telluride quantum wells [9]. There is an energy gap in a TI, however, the surface states are gapless. A typical feature of a TI-BI transition is a band inversion, when the control parameter (e.g. electric field) attains a critical value. We mention in passing that there are other 2D gapped Dirac materials isostructural with graphene: germanene $(\mathrm{Ge})$, tinene $(\mathrm{Sn})$ or Indiene (In) [10]. There are experimental and theoretical studies of these systems [8-27].

Information theoretical concepts have already been applied in studying quantum phase transitions [28-34]. TPT has also been analyzed by Shannon, Rényi and relative information and uncertainty relations [35-39]. Recently, we have shown that RényiFisher entropy product is a good marker of TPT [40]. All these previous investigations were done in either the position or the momentum space. Here we make an extension and broaden our analysis to the phase space. We select the phase-space Fisher information (PSFI), a quantity that has received almost no attention [3]. It is now shown that PSFI marks TPT since the electron-hole PSFI takes its minimum at the charge neutrality points. We also derive bounds for PSFI and introduce a topological quantum number.

The paper is organized as: TPT in 2D gapped Dirac crystals is summarized in the following section. PSFI of silicene is presented in Sect. 3, while in Sect. 4 results for the variance are shown and bounds for the PSFI are derived. The last section contains conclusions.

\section{Low energy eigenvalue problem of 2D crystals}

The low energy effective Hamiltonian can be written [18] around the Dirac point as

$$
H_{\xi s}=v_{F}\left(\sigma_{x} p_{x}-\xi \sigma_{y} p_{y}\right)-\frac{1}{2} \xi s \Delta_{\mathrm{so}} \sigma_{z}+\frac{1}{2} \Delta_{z} \sigma_{z}
$$

The parameter $\xi$ is applied to mark the inequivalent corners $K(\xi=1)$ and $K^{\prime}(\xi=-1)$ of the Brillouin zone. $\sigma_{j}$ are the Pauli matrices and $v_{F}=10^{6} \mathrm{~ms}^{-1}$ denotes the Fermi velocity of the Dirac fermions. The monolayer silicene film is placed in the external fields. The electric fields $\mathcal{E}_{z}$ produces a potential difference $\Delta_{z}=l \mathcal{E}_{z}$ between the sub-lattices with a separation $l$. The spin-orbit interaction leads to the band gap $\Delta_{\text {so }}$ and therefore the Dirac fermions possess a mass [10,19-21]. Up (down) spin is marked by $s= \pm 1$. Perpendicularly to the silicene plane a magnetic field $B$ acts. Using the Landau gauge, $\mathbf{A}=(-B y, 0,0)$ in the momentum operator $\hat{\mathbf{p}}_{i} \rightarrow \hat{\mathbf{p}}_{i}+q \mathbf{A}_{i}$, the low energy Hamiltonian takes the form

$$
\hat{H}_{s \xi}=\left(\begin{array}{cc}
\Delta_{s \xi} & v_{F}\left[\xi\left(\hat{p}_{x}-\frac{e}{c} B y\right)-i \hat{p}_{y}\right] \\
v_{F}\left[\xi\left(\hat{p}_{x}-\frac{e}{c} B y\right)+i \hat{p}_{y}\right] & -\Delta_{s \xi}
\end{array}\right),
$$

where

$$
\Delta_{s \xi}=\frac{1}{2}\left(\Delta_{z}-s \xi \Delta_{\mathrm{so}}\right)
$$


There is a TPT: we have the phase TI if $\left|\Delta_{z}\right|<\Delta_{\text {so }}$ and the phase BI when $\left|\Delta_{z}\right|>\Delta_{\text {so }}$. The effective gap $\left|\Delta_{s \xi}\right|$ disappears at CNP [18,24-26,35-38]. The eigenvalue equation of the model is [10,19-21]

$$
\hat{H} \psi=\epsilon \psi,
$$

where

$$
\psi=\left(\begin{array}{l}
\psi_{A} \\
\psi_{B}
\end{array}\right)
$$

are associated with the sublattices $A$ and $B$. The solution of Eq. (4) takes the form

$$
\epsilon_{n}^{s \xi}= \begin{cases}\operatorname{sgn}(n) \sqrt{|n| \hbar^{2} \omega^{2}+\Delta_{s \xi}^{2}}, & n \neq 0 \\ -\xi \Delta_{s \xi}, & n=0\end{cases}
$$

and

$$
|n\rangle_{s \xi}=\left(\begin{array}{c}
-i A_{n}^{s \xi}|| n\left|-\xi_{+}\right\rangle \\
B_{n}^{s \xi}|| n\left|-\xi_{-}\right\rangle
\end{array}\right)
$$

$|n\rangle$ stands for the orthonormal Fock eigenstates of the harmonical oscillator having $n=0, \pm 1, \pm 2, \ldots \omega=v_{F} \sqrt{2 e B / \hbar}$ is the cyclotron frequency and $\xi_{ \pm}=(1 \pm \xi) / 2$. The coefficients $A_{n}^{s \xi}$, and $B_{n}^{s \xi}$ have the form [24-26]

$$
\begin{gathered}
A_{n}^{s \xi}= \begin{cases}\operatorname{sgn}(n) \sqrt{\frac{\left|E_{n}^{s \xi}\right|+\operatorname{sgn}(n) \Delta_{s \xi}}{2\left|E_{n}^{s \xi}\right|},} & n \neq 0, \\
\xi_{-}, & n=0,\end{cases} \\
B_{n}^{s \xi}= \begin{cases}\sqrt{\frac{\left|E_{n}^{s \xi}\right|-\operatorname{sgn}(n) \Delta_{s \xi}}{2\left|E_{n}^{s \xi}\right|}}, & n \neq 0, \\
\xi_{+}, & n=0\end{cases}
\end{gathered}
$$

As we are interested in the density, the plane-wave $e^{i k x}$ can be discarded and we have

$$
\langle y \mid n\rangle=\frac{\omega^{1 / 4}}{\sqrt{2^{n} n ! \sqrt{\pi}}} e^{-\omega y^{2} / 2} H_{n}(\sqrt{\omega} y)
$$

in the position space, where $H_{n}$ are the Hermite polynomials of degree $n$. The density arising from (7) can be written as

$$
\rho_{n}^{s \xi}(y)=\left(A_{n}^{s \xi}\right)^{2}\left|\left\langle y|| n \mid-\xi_{+}\right\rangle\right|^{2}+\left(B_{n}^{s \xi}\right)^{2}\left|\left\langle y|| n \mid-\xi_{-}\right\rangle\right|^{2}
$$

The Fock states in momentum space read as

$$
\gamma_{n}^{s \xi}\left(p_{y}\right)=\left(A_{n}^{s \xi}\right)^{2}\left|\left\langle p_{y}|| n \mid-\xi_{+}\right\rangle\right|^{2}+\left(B_{n}^{s \xi}\right)^{2}\left|\left\langle p_{y}|| n \mid-\xi_{-}\right\rangle\right|^{2}
$$


where

$$
\left\langle p_{y} \mid n\right\rangle=\frac{\omega^{-1 / 4}}{\sqrt{2^{n} n ! \sqrt{\pi}}} e^{-p_{y}^{2} /(2 \omega)} H_{n}\left(p_{y} / \sqrt{\omega}\right) .
$$

Note that the position and momentum space densities have almost the same form.

\section{Phase-space Fisher information}

Fisher information (FI) [1] is given by

$$
I=\int \frac{\left[p^{\prime}(x)\right]^{2}}{p(x)} d x,
$$

where the probability density function (PDF) $p(x)$ fulfills the condition

$$
\int p(x) d x=1
$$

The Cramer-Rao inequality [2] relates FI to the variance

$$
\operatorname{Var}[x] \geq I^{-1}
$$

That is, a narrower PDF leads to a larger FI. Therefore, FI is considered to be a local measure, describing the 'tightness' of a distribution. We have equality in the relation (15) in case of a normal distribution. As FI is a gradient functional of the PDF, it is sensitive to local rearrangements of the density. In a phase transition we have always a sudden change in the properties of the quantum systems. So, FI incorporates information about the localization and shape of the density.

In the knowledge of the position $\left(\rho_{n}^{s \xi}(x)\right)$ and momentum $\left(\gamma_{n}^{s \xi}(p)\right)$ PDFs, the position-space $\left(I_{\rho_{n}^{s \xi}}\right)$ and momentum-space $\left(I_{\gamma_{n}^{s \xi}}\right)$ FI can be determined. Applying expressions (10), (11) and (13) we can immediately derive the relation

$$
\omega^{-1} I_{\rho_{n}^{s \xi}}=\omega I_{\gamma_{n}^{s \xi}}
$$

In this paper the main emphasis is on the PSFI. Earlier it was proved that the sum of the position and momentum space Fisher information is an upper bound of the PSFI [3]. In the following this sum will be referred as PSFI. In our case it takes the form

$$
I_{n}^{s \xi}=\omega^{-1} I_{\rho_{n}^{s \xi}}+\omega I_{\gamma_{n}^{s \xi}} .
$$

Note that the position part is divided by $\omega$, while the momentum part is multiplied by $\omega$ for dimensional reasons [41]. (Unit mass for the oscillator is supposed.) Utilizing Eq. (16) PSFI (17) can also be written as

$$
I_{n}^{s \xi}=2 \omega^{-1} I_{\rho_{n}^{s \xi}}=2 \omega I_{\gamma_{n}^{s \xi}}
$$



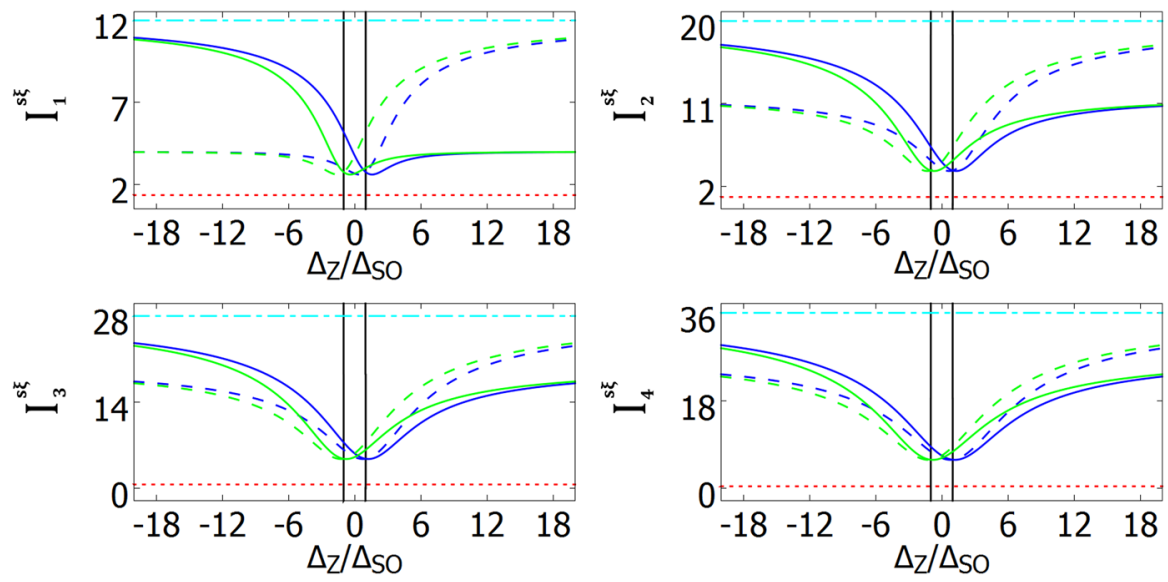

Fig. 1 Phase-space Fisher information $I_{n}^{s \xi}$ against $\Delta_{z} / \Delta_{s o}$ for the Landau levels $|n|=1,2,3,4$ and $\xi=1$. Blue (green) curves stand for spin up (down). Solid lines for $n$ positive and dash lines for $n$ negative. Upper (lower) limit is denoted by cyan dashed (red point) lines (Color figure online)

and is shown in Fig. 1. for silicene as a function of $\Delta_{z} / \Delta_{\text {so }}$ for Landau levels $|n|=$ $1,2,3,4$ for $\xi=1$. We can see that electrons and holes (separately) incorporate information about the different phases. The PSFI curves for electrons and holes cross each other when there is a change from the TI to the BI phase, that is, we gain information about TPT when we consider both (electrons and holes ) simultaneously. Therefore, we calculated the sum of PSFI for electrons and holes in silicene

$$
\hat{I}_{n}^{s \xi}=I_{n}^{s \xi}+I_{-n}^{s \xi}
$$

and plotted in Fig. 2 versus $\Delta_{z} / \Delta_{s o}$. There are minima at the charge neutrality points (CPN) $\Delta_{z}=\Delta_{s o}\left(\Delta_{z}=-\Delta_{s o}\right)$ for Landau levels $|n|=0,1,2,3,4$ both for spin up and down cases. One can also notice that the slopes of the curves have the same sign in the phase BI $\left(\left|\Delta_{z}\right|>\Delta_{\text {so }}\right)$ and have different sign in the phase TI $\left(\left|\Delta_{z}\right|<\Delta_{\text {so }}\right)$. Taking advantage of this fact we can construct a topological quantum number (TPQ):

$$
C_{n s \xi}^{1}=\operatorname{sgn}\left(\frac{\partial \hat{I}_{n}^{s \xi}}{\partial \Delta_{z}} \frac{\partial \hat{I}_{n}^{-s \xi}}{\partial \Delta_{z}}\right)
$$

This quantity can be considered a topological charge (TC)

$$
C_{n s \xi}^{1}\left(\Delta_{z}\right)=\left\{\begin{array}{cc}
1,\left|\Delta_{z}\right|>\Delta_{S O} & B I \\
-1,\left|\Delta_{z}\right|<\Delta_{S O} & T I
\end{array}\right.
$$

which is capable to identify different phases like the Chern number. 

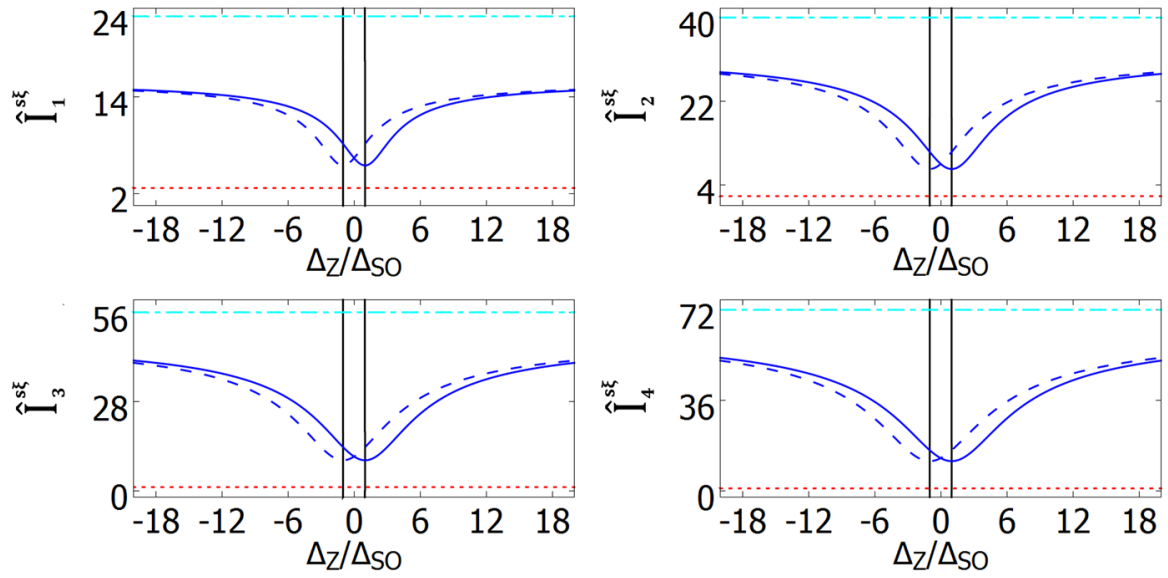

Fig. 2 Phase-space Fisher information for electrons and holes $\hat{I}_{n}^{s \xi}$ against $\Delta_{z} / \Delta_{\text {so }}$ for $\xi=1$ and Landau levels $|n|=1,2,3,4$. Upper (lower) limit is denoted by cyan dashed (red point) lines (Color figure online)

\section{Variance and bounds for the phase-space Fisher information}

The variance of the $\operatorname{PDF} \rho$ is given by

$$
\operatorname{Var}(x)=\sigma_{x}^{2}=\int_{-\infty}^{+\infty}(x-\mu)^{2} \rho(x) d x=\left\langle(x-\mu)^{2}\right\rangle,
$$

where

$$
\mu=\int_{-\infty}^{+\infty} x \rho(x) d x=\langle x\rangle
$$

It can also be written as

$$
\operatorname{Var}(x)=\left\langle x^{2}\right\rangle-\mu^{2} .
$$

Taking into account that the PDF studied here are even functions [see Eg. (10)] the expectation value of $x$ disappears: $\langle x\rangle=0$. Consequently, the variance takes the form

$$
\operatorname{Var}(x)=\left\langle x^{2}\right\rangle
$$

Recall now the well-known expression for the variance of the linear harmonic oscillator

$$
\left\langle x^{2}\right\rangle_{n}=\frac{\hbar}{\omega}\left(n+\frac{1}{2}\right)
$$

in the $n$th quantum state with $\hbar=1$ in the rest of the paper. (It can be found in most textbooks on quantum mechanics or can be derived from the recursion relation on Hermite polynomials. Unit mass for the oscillator is supposed.) Applying the virial theorem the variance in momentum space can be written as 


$$
\left\langle p^{2}\right\rangle_{n}=\omega\left(n+\frac{1}{2}\right)
$$

The variance for the PDF (10) has the form

$$
\left\langle x^{2}\right\rangle_{n}^{s \xi}=\frac{1}{\omega}\left[\left(A_{n}^{s \xi}\right)^{2}\left(|n|-\xi_{+}+\frac{1}{2}\right)+\left(B_{n}^{s \xi}\right)^{2}\left(|n|-\xi_{-}+\frac{1}{2}\right)\right]
$$

Utilizing Eqs. (10), (11), (26) and (27) we can obtain

$$
\omega\left\langle x^{2}\right\rangle_{n}^{s \xi}=\omega^{-1}\left\langle p^{2}\right\rangle_{n}^{s \xi}
$$

If $n=0$ we obtain $\left\langle x^{2}\right\rangle_{0}^{s \xi}=\frac{1}{2 \omega}$. Taking into account that $\left(A_{n}^{s \xi}\right)^{2}=1-\left(B_{n}^{s \xi}\right)^{2}$, we are led to

$$
\left\langle x^{2}\right\rangle_{n}^{s \xi}=\frac{1}{\omega}\left[|n|+\xi\left(\left(B_{n}^{s \xi}\right)^{2}-\frac{1}{2}\right)\right] .
$$

It can be seen from Eq. (8) that $0<\left(B_{n}^{s \xi}\right)^{2}<1$ if $n \neq 0$. Therefore, we obtain the relations

$$
\frac{1}{\omega}\left(|n|-\frac{1}{2}\right) \leq\left\langle x^{2}\right\rangle_{n}^{s \xi} \leq \frac{1}{\omega}\left(|n|+\frac{1}{2}\right)
$$

and

$$
\omega\left(|n|-\frac{1}{2}\right) \leq\left\langle p^{2}\right\rangle_{n}^{s \xi} \leq \omega\left(|n|+\frac{1}{2}\right) .
$$

Taking into account the Cramer-Rao inequality (15) we arrive at the inequality

$$
\frac{\omega}{|n|+\frac{1}{2}} \leq I_{\rho_{n}^{s \xi}}
$$

The corresponding inequality for the momentum space FI has the form

$$
\frac{1}{\omega\left(|n|+\frac{1}{2}\right)} \leq I_{\gamma_{n}^{s \xi}} .
$$

Turn now to Stam uncertainty principle

$$
I_{\rho_{n}^{s \xi}} \leq 4 \sigma_{\gamma_{n}^{s \xi}}
$$

in position space and

$$
I_{\gamma_{n}^{s \xi}} \leq 4 \sigma_{\rho_{n}^{s \xi}}
$$

in momentum space. Combining inequalities (31), (32), (35) and (36) we obtain upper bounds to the FI:

$$
I_{\rho_{n}^{s \xi}} \leq 4 \omega\left(|n|+\frac{1}{2}\right)
$$


and

$$
I_{\gamma_{n}^{s \xi}} \leq \frac{4}{\omega}\left(|n|+\frac{1}{2}\right) .
$$

Inequalities (33), (34), (37) and (38) can be put together as

$$
\frac{\omega}{|n|+\frac{1}{2}} \leq I_{\rho_{n}^{s \xi}} \leq 4 \omega\left(|n|+\frac{1}{2}\right)
$$

and

$$
\frac{1}{\omega\left(|n|+\frac{1}{2}\right)} \leq I_{\gamma_{n}^{s \xi}} \leq \frac{4}{\omega}\left(|n|+\frac{1}{2}\right) .
$$

Note that the bounds do not depend on $s$ and $\xi$. If $n=0$ we obtain equalities in relations (39) and (40): $I_{\rho_{0}^{s \xi}}=2 \omega$ and $I_{\gamma_{0}^{s \xi}}=2 / \omega$.

Equations (16), (17), (18), (19), (33) and (34) lead to lower and upper bounds for PSFI

$$
\frac{2}{|n|+\frac{1}{2}} \leq I_{n}^{s \xi} \leq 8\left(|n|+\frac{1}{2}\right)
$$

and

$$
\frac{4}{|n|+\frac{1}{2}} \leq \hat{I}_{n}^{s \xi} \leq 16\left(|n|+\frac{1}{2}\right) .
$$

$\hat{I}_{n}^{s \xi}$ stands for the electron plus hole PSFI sum. These limits are also presented in Figs. 1. and 2. as horizontal lines for $n=1,2,3,4$.

The plot of the PSFI of electrons plus holes hints that there is a delocalization in the vicinity of CNP-s. However, the variance sum for electrons and holes does not support this assumption.

To obtain further knowledge on the Cramer-Rao inequality (15) define the differences

$$
J_{\rho_{n}^{s \xi}}=\operatorname{Var}_{\rho_{n}^{s \xi}}(x)-\left(I_{\rho_{n}^{s \xi}}\right)^{-1}
$$

and

$$
J_{\gamma_{n}^{s \xi}}=\operatorname{Var}_{\gamma_{n}^{s \xi}}(p)-\left(I_{\gamma_{n}^{s \xi}}\right)^{-1}
$$

The phase-space differences are

$$
J_{n}^{s \xi}=\omega J_{\rho n}^{s \xi}+\omega^{-1} J_{\gamma_{n}^{s \xi}}
$$

and

$$
\hat{J}_{n}^{s \xi}=J_{n}^{s \xi}+J_{-n}^{s \xi} .
$$

The latter expression for the combined difference for electrons and holes is shown in Fig. 3. There are minima at CNP both for spin up and down cases. The slopes of the curves have the same (opposite) sign in the phase BI (TI) that makes it possible to introduce another form of TQN 

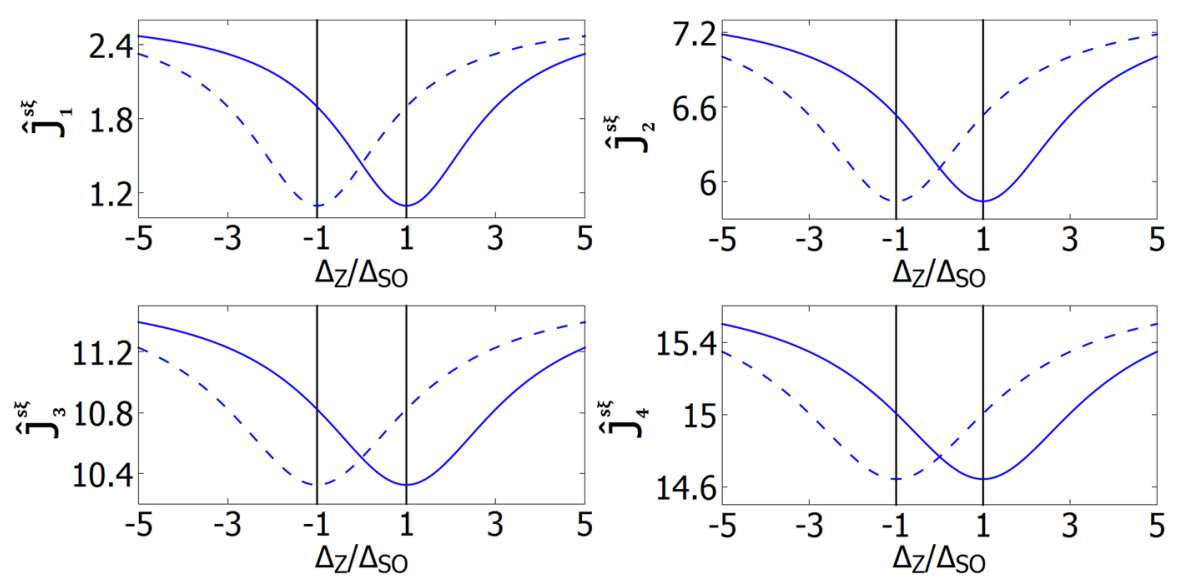

Fig. 3 Combined difference $\hat{J}_{n}^{s \xi}$ for electrons and holes against $\Delta_{z} / \Delta_{\text {so }}$ for Landau levels $|n|=1,2,3,4$ for spin up (solid lines) and spin down (dashed lines) in the valley $\xi=1$

$$
C_{n s \xi}^{2}=\operatorname{sgn}\left(\frac{\partial \hat{J}_{n}^{s \xi}}{\partial \Delta_{z}} \frac{\partial \hat{J}_{n}^{-s \xi}}{\partial \Delta_{z}}\right) .
$$

This quantity can also be identified as the TC

$$
C_{n s \xi}^{2}\left(\Delta_{z}\right)=\left\{\begin{array}{cc}
1,\left|\Delta_{z}\right|>\Delta_{S O} & B I \\
-1,\left|\Delta_{z}\right|<\Delta_{S O} & T I
\end{array}\right.
$$

Turn now to Stam uncertainty principle [(35) and (36)] and define the position-

$$
Z_{\rho_{n}^{s \xi}}=I_{\rho_{n}^{s \xi}}-4 \sigma_{\gamma_{n}^{s \xi}} \leq 0
$$

and momentum-space

$$
Z_{\gamma_{n}^{s \xi}}=I_{\gamma_{n}^{s \xi}}-4 \sigma_{\rho_{n}^{s \xi}} \leq 0
$$

differences. One can easily prove that

$$
\omega^{-1} Z_{\rho_{n}^{s \xi}}=\omega Z_{\gamma_{n}^{s \xi}}
$$

The corresponding phase-space difference is defined as

$$
Z_{n}^{s \xi}=\omega^{-1} Z_{\rho_{n}^{s \xi}}+\omega Z_{\gamma_{n}^{s \xi}} \leq 0 .
$$

It is worth summing the differences of electrons and holes

$$
\hat{Z}_{n}^{s \xi}=Z_{n}^{s \xi}+Z_{-n}^{s \xi} \leq 0 .
$$

This quantity is presented in Fig. 4 against $\Delta_{z} / \Delta_{s o}$ for Landau levels $|n|=1,2,3,4$ in the valley $\xi=1$. The curves have minima at the CNP and the slopes of the curves 

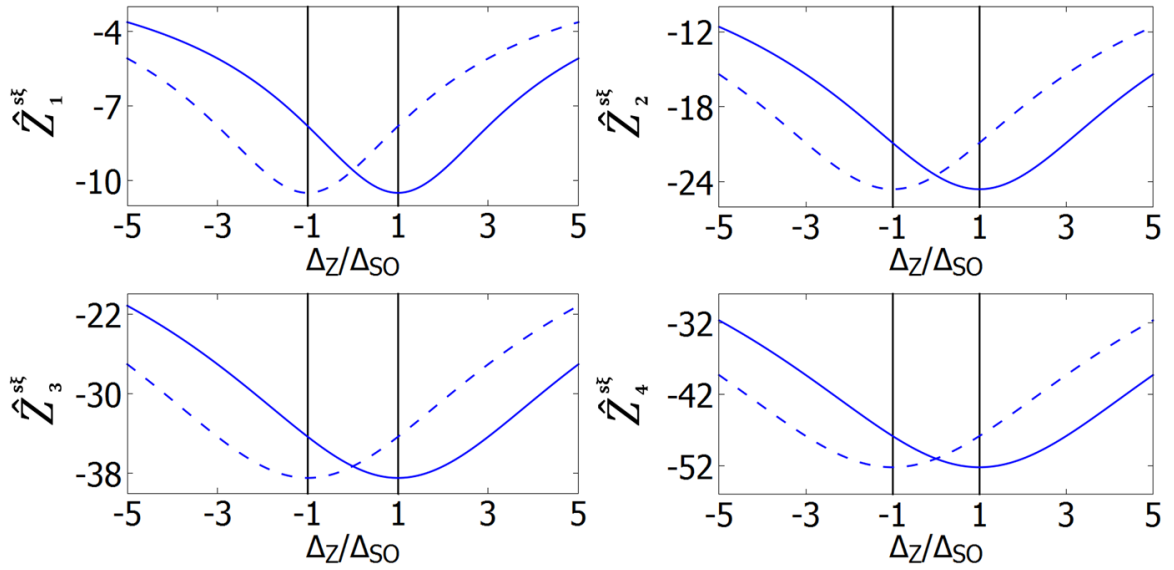

Fig. 4 Combined difference $\hat{Z}_{n}^{s \xi}$ for electrons and holes against $\Delta_{z} / \Delta_{\text {so }}$ for Landau levels $|n|=1,2,3,4$ for spin up (solid lines) and spin down (dashed lines) in the valley $\xi=1$

have the same (different) sign in the phase BI (TI). This result provides for defining TQN in another form

$$
C_{n s \xi}^{3}=\operatorname{sgn}\left(\frac{\partial \hat{Z}_{n}^{s \xi}}{\partial \Delta_{z}} \frac{\partial \hat{Z}_{n}^{-s \xi}}{\partial \Delta_{z}}\right) .
$$

TC can be now formalized as

$$
C_{n s \xi}^{3}\left(\Delta_{z}\right)=\left\{\begin{array}{cc}
1,\left|\Delta_{z}\right|>\Delta_{S O} & B I \\
-1,\left|\Delta_{z}\right|<\Delta_{S O} & T I
\end{array}\right.
$$

\section{Conclusions}

We have analyzed how PSFI characterize TPT in silicene, a 2D Dirac gapped material. The PSFI of electrons plus holes exhibit minimum at the CNP $\Delta_{z}=\Delta_{s o}\left(\Delta_{z}=-\Delta_{s o}\right)$ for spin up (down) case. Moreover, a TQN capable to identify the different phases has been defined utilizing combined PSFI in three forms. Upper and lower bounds for PSFI have been derived. We have analyzed the difference of the variance and the inverse Fisher measures. It has turned out that the sum of the differences exhibits minima at CPN. TQN can be defined utilizing this quantity. Stam inequality has also turned out to be useful in detecting TPT and defining a TQN. In summary, PSFI has been found to be a new marker of topological phase transitions.

Acknowledgements Open access funding provided by University of Debrecen (DE). The work was supported by the Junta de Andalucía Project FQM-381 and the National Research, Development and Innovation Fund of Hungary, financed under the 123988 funding scheme and the EU-funded Hungarian Grant EFOP3.6.2-16-2017-00005. 
Open Access This article is distributed under the terms of the Creative Commons Attribution 4.0 International License (http://creativecommons.org/licenses/by/4.0/), which permits unrestricted use, distribution, and reproduction in any medium, provided you give appropriate credit to the original author(s) and the source, provide a link to the Creative Commons license, and indicate if changes were made.

\section{References}

1. R.A. Fisher, Proc. Camb. Philos. Soc. 22, 700 (1925)

2. B.R. Frieden, Physics from Fisher Information. A Unification (Cambridge University Press, Cambridge, 1998)

3. I. Hornyák, Á. Nagy, Chem. Phys. Lett. bf 437, 132 (2007)

4. C.-C. Liu, H. Jiang, Y. Yao, Phys. Rev. B 84, 195430 (2011)

5. C.-C. Liu, W. Feng, Y. Yao, Phys. Rev. Lett. 107, 076802 (2011)

6. M. Ezawa, J. Physical Soc. Japan 84, 121003 (2015)

7. M. Xu, T. Liang, M. Shi, H. Chen, Chem. Rev. 113, 766 (2013)

8. C.L. Kane, E.J. Mele, Phys. Rev. Lett. 95, 226801 (2005)

9. B. Andrei Bernevig, Taylor L. Hughes, Shou-Cheng Zhang, Science 314, 1757-1761 (2006)

10. W.-F. Tsai, C.-Y. Huang, T.-R. Chang et al., Nat. Commun. 4, 1500 (2013)

11. K. Takeda, K. Shiraishi, Phys. Rev. B 50, 14916 (1994)

12. G.G. Guzman-Verri, L. Lew Yan, Phys. Rev. B 76, 075131 (2007)

13. P. Vogt et al., Phys. Rev. Lett. 108, 155501 (2012)

14. B. Augray, A. Kara, S.B. Vizzini, H. Oughaldou, C. LéAndri, B. Ealet, G. Le Lay, Appl. Phys. Lett. 96, $183102(2010)$

15. B. Lalmi, H. Oughaddou, H. Enriquez, A. Kara, S.B. Vizzini, B.N. Ealet, B. Augray, Appl. Phys. Lett. 97, 223109 (2010)

16. A. Feurence, R. Friedlein, T. Ozaki, H. Kawai, Y. Wang, Y.Y. Takamura, Phys. Rev. Lett. 108, 245501 (2012)

17. P.E. Padova et al., Appl. Phys. Lett. 96, 261905 (2010)

18. M. Tahir, U. Schwingenschlögl, Sci. Rep. 3, 1075 (2013)

19. N.D. Drummond, V. Zólyomi, V.I. Fal'ko, Phys. Rev. B 85, 075423 (2012)

20. C.C. Liu, W. Feng, Y. Yao, Phys. Rev. Lett. 107, 076802 (2011)

21. C.C. Liu, H. Jiang, Y. Yao, Phys. Rev. B 84, 195430 (2011)

22. S. Trivedi, A. Srivastava, R. Kurchania, J. Comput. Theor. Nanosci. 11, 1-8 (2014). https://doi.org/10. $1166 /$ jctn.2014.3428

23. B. van den Broek et al., 2D Mater. 1, 021004 (2014). https://doi.org/10.1088/2053-1583/1/2/021004

24. L. Stille, C.J. Tabert, E.J. Nicol, Phys. Rev. B 86, 195405 (2012)

25. C.J. Tabert, E.J. Nicol, Phys. Rev. Lett. 110, 197402 (2013)

26. C.J. Tabert, E.J. Nicol, Phys. Rev. B 88, 085434 (2013)

27. M. Ezawa, New J. Phys. 14, 033003 (2012)

28. S.J. Gu, Int. J. Mod. Phys. B 24, 4371 (2010)

29. L.A. Wu, M.S. Sarandy, D.A. Lidar, L.J. Sham, Phys. Rev. A 74, 052335 (2006)

30. Á. Nagy, M. Calixto, E. Romera, J. Chem. Theory Comput. 9, 1068 (2013)

31. Á. Nagy, E. Romera, Phys. Rev. A 88, 042515 (2013)

32. S.J. Gu, Chem. Phys. Lett. 26, 026401 (2009)

33. E. Romera, Á. Nagy, Phys. Lett. A 377, 3098 (2013)

34. Á. Nagy, E. Romera, Europhys. Lett. bf 109, 60002 (2015)

35. E. Romera, M. Calixto, EPL 111, 37006 (2015)

36. M. Calixto, E. Romera, J. Stat. Mech. P06029 (2015)

37. E. Romera, M. Calixto, J. Phys. Condens. Matter 27, 175003 (2015)

38. M. Calixto, E. Romera, EPL 109, 40003 (2015)

39. J.C. Bolívar, E. Romera, Phys. Lett. A 381, 1753 (2017) 
40. J.C. Bolívar, Á. Nagy, E. Romera, Physica A 498, 66 (2018)

41. F. Pennini, A. Plastino, Phys. Lett. A bf 349, 15 (2006)

Publisher's Note Springer Nature remains neutral with regard to jurisdictional claims in published maps and institutional affiliations. 